\section{PERCEPÇÕES DE PESQUISADORES BRASILEIROS SOBRE O ACESSO ABERTO À LITERATURA CIENTÍFICA}

\author{
Ariadne Chloe Mary Furnival \\ Nelson Sebastian Silva-Jerez $z^{* *}$
}

RESUMO:

A pesquisa se propôs explorar como várias dimensões do acesso aberto à literatura científica são percebidas por pesquisadores brasileiros, identificando também seus hábitos de publicação, uso e citação de fontes em acesso aberto. A abordagem metodológica foi quantitativa, com aplicação de um questionário a 643 doutores permanentes distribuídos nas universidades brasileiras, oriundos de todos as áreas disciplinares. O questionário foi elaborado com auxílio da plataforma livre LimeSurvey, sendo composto por 29 questões fechadas. Foram recebidos questionários respondidos por pesquisadores oriundos de 3 I Institutos de Ensino Superior e Institutos de Pesquisa no Brasil. A estratificação por área mostrou uma distribuição relativamente equilibrada entre os campos de conhecimento, com a maior porcentagem sendo das Ciências da Saúde (21\%), seguido pelas Ciências Exatas e da Terra (20,1\%), e a menor porcentagem, de I, I\%, de Linguística, Letras e Artes. Os resultados apontaram que a grande maioria dos pesquisadores respondentes detém conhecimento sobre, e apoiam os princípios e algumas ações de acesso aberto, principalmente em relação à publicação de revistas acesso aberto, o que reflete também nos seus hábitos de uso e citação dessas fontes. Comparativamente, o conhecimento e uso de repositórios é menor, e parece haver uma certa confusão em relação ao status de copyright dos artigos arquivados em repositórios. Ficou evidente que os pesquisadores acreditam que somente os postprints deveriam ser disponibilizados em repositórios. O desconhecimento identificado acerca da compatibilidade entre a publicação em revistas "convencionais", de assinatura e o arquivamento do mesmo artigo em repositórios em acesso aberto aponta a necessidade de maiores iniciativas informativas para esclarecer esses aspectos.

Palavras-chave: Acesso aberto. Pesquisadores. Opiniões. Publicação. comunicação científica.
* Doutora em Política Científica e Tecnológica pla Universidade Estadual de Campinas, Brasil. Professora Associada do Departamento de Ciência da Informação da Universidade Federal de São Carlos, Brasil.

E-mail: chloe@ufscar.br.

** Mestre em Ciência da Informação pela Universidade Estadual Paulista Julio de Mesquita Filho, Brasil. Doutorando no Programa de Pós-Graduação em Ciência da Informação da Universidade Estadual Paulista Julio de Mesquita Filho, Brasil.

E-mail: agentecounter-assuntosacademicos@yahoo.com.br.

\section{INTRODUÇÃO}

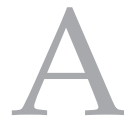

publicação científica em acesso aberto faz parte de um cenário mais amplo em prol da abertura ao conhecimento em geral (acesso aberto, dados abertos, recursos educacionais abertos, software livre, licenças abertas) e constitui essencialmente um movimento na direção da informação e conhecimento como um bem público. Como observa Benkler (2010, p. 217, tradução nossa), "Nos últimos anos, uma coalizão diversa de movimentos, atores políticos e econômicos, ONGs, cientistas e outros acadêmicos, começaram 
a se reunir em torno da ideia ou slogan, 'acesso ao conhecimento' - A2K.". No contexto do acesso aberto (no inglês open access) à literatura científica, este surge da convergência de dois fatores: um é a antiga boa-vontade de investigadores e cientistas publicarem os resultados de suas pesquisas em revistas científicas, sem qualquer remuneração, apenas em prol da investigação e difusão do conhecimento; e o outro é o crescente aumento do acesso à internet (RODRIGUES, 2004; SUBER, 2012). O benefício público que as duas possibilitam é a distribuição eletrônica, em uma escala mundial, da literatura científica com revisão pelos pares, de forma gratuita e, se for em acesso aberto, sem restrições de acesso e reuso aos interessados. É reconhecido que a eliminação de barreiras de acesso à literatura científica ajudará a acelerar a pesquisa e evolução da ciência, enriquecer a educação e subsidiar a tomada de decisões de políticas públicas.

Suber (2010, p.1), um dos idealizadores do movimento de Acesso Aberto, indaga: "Por que não há mais autores que aproveitem da revolução de acesso para alcançar mais leitores?". Tal questionamento alude ao consenso na literatura de que os desafios técnicos para alcançar o acesso aberto têm sido resolvidos, mas aqueles relacionados às ações visando à mudança de hábitos e incentivos para a publicação em acesso aberto continuam sendo os mais formidáveis a superar. Mas é verdade afirmar que, se a comunidade científica não aderir ao acesso aberto - da perspectiva de disponibilizar sua produção em canais de acesso aberto (seja pela "via dourada", ou pela "via verde") - então este pode estar fadado a fracassar. Estudos de várias partes do mundo demonstram que são justamente os pesquisadores o grupo que tem sido mais difícil a convencer a "abraçar a causa" do acesso aberto, tendo sido surpreendentemente resistentes em adotar práticas e hábitos para acelerar a sua difusão e consolidação:

\footnotetext{
No entanto, os próprios pesquisadores, têm sido os mais difíceis a motivar para agir. (...) em geral os pesquisadores tem sido lentos em agir de forma que possa concretizar 0 acesso aberto, constituindo um retardador significativo ao progresso deste, já que sua implementação fica principalmente nas mãos da própria comunidade científica (SWAN, 2006a, p.65; tradução nossa).
}

Nesse sentido, os indicadores são desapontadores: apenas em torno de $30 \%$ do total dos 2,5 milhões de artigos científicos publicados no mundo anualmente está disponível em canais acesso aberto (BJÖRK et al., 2010; HAJJEM et al., 2005). Harnard (2006) acrescenta que apenas $15 \%$ dos autores científicos auto-arquivam seus artigos em repositórios institucionais. Em 2007, Davis e Connolly (2007) observaram que, apesar do investimento institucional, o repositório institucional da Cornell University nos EUA estava sendo consideravelmente subutilizado pelo corpo docente dessa universidade, e de modo semelhante, Ferreira et al. (2008) reclamaram que os esforços para encorajar o arquivamento no repositório institucional da Universidade de Minho, lançado em 2003, "não surtiram os efeitos esperados" na época. Swan (2006b) relata que dos autores entrevistados nas pesquisas realizadas por ela e Brown (SWAN e BROWN, 2004; 2005), 20\% auto-arquivaram cópias num repositório de acesso aberto e $25 \%$ submeteram um artigo a uma revista acesso aberto, embora $49 \%$ tenham afirmado que tinham a intenção de submeter. Esses números, segundo Swan (2006b, p.67), não sustentam a noção de que os pesquisadores se importam mais do que tudo com fazer um impacto no seu campo de pesquisa.

Tudo isso está em nítido contraste com o crescimento contínuo do número de repositórios de acesso aberto sendo implantados mundialmente. Um diretório que os cadastra, o OpenDOAR, mostra que seu número (que inclui a categoria "biblioteca digital") teve um crescimento de quase $2.500 \%$ entre 2005 e 2017 (OpenDOAR, 2017), alcançando 3.291 repositórios registrados no mês de janeiro de 2017. Ao mesmo tempo, há indicativos que o cenário de acesso aberto da via verde no Brasil ainda pode ser classificado como incipiente: apesar dos 91 repositórios brasileiros cadastrados no OpenDOAR, Prost e Schöpfel (2014) não conseguiram identificar repositórios de acesso aberto na América do Sul contendo mais que 10 mil itens para incluir no seu levantamento, um cenário que contrasta com o estado comparativamente saudável da via dourada brasileira, que deve-se principalmente ao sucesso do SciELO, com seus 279 títulos correntes de revistas acesso aberto (do SciELO Brasil, exclusivamente). 
Cabe perguntar se os pesquisadores da comunidade científica no Brasil percebem as potenciais vantagens ${ }^{1}$ de disseminar suas publicações em canais de acesso aberto, e por que existe uma relativa baixa adesão à via verde para a disseminação e publicação em acesso aberto. Mas ainda existem poucas pesquisas no Brasil que exploram as opiniões e percepções da comunidade científica brasileira sobre o acesso aberto.

Propôs-se aqui, então, levantar as opiniões de uma amostra de pesquisadores da comunidade científica brasileira, em relação à crescente disseminação e publicação em acesso aberto. Mais especificamente, buscou-se identificar os fatores que afetam a sua aceitação ou sua resistência em publicar suas pesquisas nas revistas acesso aberto ou depositar cópias dos seus trabalhos em repositórios de acesso aberto, além de mapear aspectos do comportamento informacional desses pesquisadores como usuários de informação no que diz respeito ao seu acesso, uso e percepções de fontes disponíveis em acesso aberto.

Os pesquisadores se localizam nos dois pontos da cadeia da comunicação científica, como produtores do objeto do movimento de acesso aberto - a saber, conhecimento científico na forma de publicações científicas - e como usuários do mesmo, cujo livre acesso à pesquisa científica publicada constitui uma parte crucial do ciclo de pesquisa. Os pesquisadores da comunidade científica, como produtores e usuários das publicações, constituem a razão de ser do movimento Acesso Aberto.

\section{ESTUDOS DAS PERCEPÇÕES DOS CIENTISTAS SOBRE ACESSO ABERTO}

O descompasso entre os esforços em implementar o acesso aberto e os resultados alcançados, tem levado muitos autores a identificar as possíveis barreiras que inibam a adoção por pesquisadores de práticas de publicação e disseminação em canais de acesso. Swan e Brown (2005) levantaram as opiniões

Ioje existe um corpo substancial de pesquisa bibliométrica que comprova a existência de uma "Vantagem de citação de acesso aberto" (Open Access Citation Advantage) - a OACA. Veja "The open citation project" em http://opcit.eprints.org/oacitation-biblio.html de pesquisadores sobre o acesso aberto e uma das perguntas feitas foi sobre o que pensam a respeito das políticas que os obrigariam a depositar cópias (os chamados mandatos) dos seus trabalhos em repositórios de acesso aberto, advindos das instituições ou das agências de fomento. A grande maioria disse que cumpriria com tais mandatos voluntariamente, $14 \%$ cumpriria com relutância e apenas 5\% disse que não cumpriria com o mandato. Mas a realidade não reflete tal "vontade": no máximo, 20\% auto-arquiva e $25 \%$ publica em revistas acesso aberto. Em outra pesquisa, realizada por Davies (2011) com um pequeno grupo de 82 químicos e economistas de 11 universidades no Reino Unido, os pesquisadores entrevistados pareceram não ter conhecimento do mandato de acesso aberto da suas respectivas instituições: de um grupo de 54 pesquisadores cujas universidades têm mandatos, apenas 7 entrevistados tinham certeza desse fato. Do modo semelhante, dos 65 pesquisadores cujas agências de fomento operam com um mandato, apenas 14 afirmaram que esses mandatos existiam. Sendo que a maioria dos entrevistados nesse estudo já estava engajados com acesso aberto, a implicação é que a política institucional teve pouco impacto (Davies, 2011). Um entrevistado alegou que se soubesse da existência de tal tipo de mandato, ele até sentiria menos vontade de se engajar com o acesso aberto.

Em outro estudo, realizado por Stone (2010) na sua própria instituição, 88\% dos respondentes eram a favor dos princípios do acesso aberto e $86 \%$ a favor do depósito dos seus artigos no repositório institucional, manifestações de apoio estas que contrastam com o apenas $29 \%$ da produção científica disponível no mesmo. Por volta de $72 \%$ dos respondentes nesse estudo eram a favor de publicar em revistas acesso aberto, embora houvessem alguns comentários demonstrando preocupação com o processo de revisão pelos pares em tal tipo de revista, preocupação esta que se repete em outros estudos.

Num estudo realizado com pesquisadores brasileiros oriundos do estado de Rio de Janeiro, Chalhub e Ribeiro (2011) reportaram que dos 28 pesquisadores estudados somente quatro (um da área de Ciências Biológicas e três de Engenharia) nunca fizeram uso de canais de acesso aberto para a divulgação dos seus resultados da pesquisa, com as revistas 
sendo o mais utilizado (79\%) dos respondentes, seguido por $25 \%$ que utilizaram repositórios. Quando perguntado se tiveram motivos para não disponibilizar seus artigos em acesso aberto, 68\% dos pesquisadores entrevistados disseram que não tiveram motivos, mas nas transcrições de algumas falas dos entrevistados naquele estudo, vários manifestaram certo receio de infringir o copyright do artigo caso decidissem arquivá-lo num repositório de acesso aberto. Esta apreensão parece ser um sentimento quase unânime entre os pesquisadores de todos os estudos das opiniões e percepções de acesso aberto entre os pesquisadores.

Swan e Brown (2005) chamaram a atenção para a falta de conhecimento entre pesquisadores dos seus direitos sobre seus próprios trabalhos em relação ao arquivamento, especificamente o fato de que $90 \%$ das editoras comerciais permitem arquivamento do postprint (Swan, 2006b, p.67). A apreensão acerca da questão de copyright é frequentemente explicada como reflexo da falta de uma leitura detalhada do acordo de transferência de copyright por parte dos pesquisadores antes de assiná-lo, mas Stone (2010) levantou um dado contrariando esse "senso comum": 68\% dos pesquisadores do estudo afirmam ler esse acordo, mas 65\% não concordam com aquilo que assinam, acreditando que os direitos deveriam ficar com o autor ou com a instituição de origem do autor. O estudo de Moore (2011) com pesquisadores da Universidade de Toronto identificou que a maior parte destes não lê os detalhes do acordo da transferência de copyright, e simplesmente o assinam. Um resultado interessante neste estudo é que da minoria dos pesquisadores $(7 \%)$ que alteram o termo de copyright, a maioria (57\%) é advinda das ciências humanas e ciências sociais.

Mundialmente, os cientistas das Exatas têm aderido ao movimento Acesso Aberto mais rapidamente, e o estudo de Toronto confirma esta tendência, com o arquivamento em repositórios de acesso aberto sendo mais comum entre os cientistas das Exatas e Engenharias, e a publicação em revistas acesso aberto mais comum para pesquisadores das humanidades. As falas dos pesquisadores referentes à sua percepção sobre estas revistas revelam as dúvidas que estes têm sobre este canal como sendo viável: ao comparálas com as "convencionais", de assinatura, os pesquisadores falaram da maior tradição, prestígio e respeito nos seus respectivos campos que estas têm quando comparadas com aquelas de acesso aberto (Moore, 2011). Uma pesquisa feita por Bongiovani, Gómez e Miguel (2012), que levantou as opiniões de 532 pesquisadores argentinos em relação à publicação em acesso aberto exclusivamente em revistas, demonstrou que estes apoiam a ideia, conhecem títulos de revistas acesso aberto nas suas respectivas áreas, mas tal apoio "retórico" não se reflete nas suas práticas de publicação, que continuam sendo nas revistas de assinatura.

Este tipo de preocupação está espelhado no estudo realizado por Park e Qin (2007) que entrevistaram 14 pesquisadores, uma mistura de doutorandos e docentes, da Universidade de Syracruse. Queriam estudar os fatores que aumentam ou diminuem a vontade dos pesquisadores publicarem em, e usarem as revistas acesso aberto somente (ou seja, o foco foi exclusivamente na via dourada). Para tanto, examinaram as percepções dos pesquisadores relativas à qualidade, reputação, disponibilidade e relevância destas revistas. Não é de se surpreender que um resultado claro da pesquisa desses autores é que a percepção da qualidade e reputação da revista afeta a disposiçãodos pesquisadores para publicar nela. Mas um paradoxo que o estudo levantou foi que por um lado, a percepção que os pesquisadores têm da disponibilidade dos artigos em acesso aberto é positiva do ponto de vista de progresso na carreira, pois dá mais visibilidade à pesquisa, e portanto, tem maior potencial para suscitar impactos. A disponibilidade percebida também afeta positivamente a percebida facilidade de uso, já que os dois fatores juntos indicam conveniência e fácil acessibilidade. Por outro lado, os autores identificaram que os pesquisadores detêm uma percepção negativa de conteúdo mais facilmente disponível, uma atitude que parece estar arraigada na sua atitude geral de terem pouca confiança na qualidade de recursos disponíveis exclusivamente pela internet (Park e Qin, 2007). É preocupante constatar nos resultados que a maioria dos seus entrevistados "não sabiam do fato que as revistas acesso aberto seguem o processo de revisão pelos pares e assim, tratam o conteúdo de revistas acesso aberto como se fosse simplesmente informação não-controlada da Internet" (Park e Qin, 2007, p.77-8). 
No Brasil, uma pesquisa qualitativa realizada entre pesquisadores das ciências agrárias oriundos de universidades do estado de São Paulo (SANTOS e MONTEIRO, 2013) também foca exclusivamente as percepções e hábitos desse grupo de pesquisadores no que tange à publicação em revistas acesso aberto. De modo semelhante daquilo que aparece nos resultados de outras pesquisas, os entrevistados equiparam estas revistas com a falta geral de qualidade e/ou a falta da revisão pelos pares, significando que esses canais não figuram nas escolhas dos pesquisadores, como visto neste trecho de uma entrevista:

\begin{abstract}
O que eu percebo destes periódicos eletrônicos é que vira e mexe eu recebo convite para liderar uma revista ou para participar de algum número específico e eu não sei como eles estão se organizando, eu admito a minha ignorância, mas de repente tem duzentas mil revistas Open Access, e como eles vão estabelecer a revisão dos artigos? Quem está revisando tudo isso? O quanto essas informações são revisadas? Queira ou não queira, essas outras revistas têm um caráter comercial, mas a gente já sabe que elas têm um corpo editorial, têm um respeito. Passam coisas ruins? Passam, mas, de forma geral, existe um controle [Pesq.6] (SANTOS; MONTEIRO, 20I3, p.43).
\end{abstract}

A pesquisa PEER - Publishing and the Ecology of European Research - (FRY, et al., 2011) levantou as atitudes e percepções de pesquisadores europeus de várias áreas disciplinares para identificar até que grau estes são cientes do acesso aberto e as diferentes formas de alcançá-lo, e os fatores (des) motivadores que influenciem sua adesão a este modo de publicar. Como nos outros estudos semelhantes, os resultados do PEER apontam para a continuada centralidade do artigo científico revisado pelos pares na carreira dos cientistas, mas que, na concepção da maioria pesquisados no estudo PEER, os artigos publicados em revistas acesso aberto são de uma qualidade inferior que aquela das tradicionais. Além disso, a maioria dos participantes do estudo associam acesso aberto com artigos em revistas acesso aberto, e uma clara minoria associou-o com o auto-arquivamento em repositórios de acesso aberto.
Os pesquisadores das Ciências Médicas, da Saúde, e das Biológicas entendem acesso aberto como sendo através da via dourada, enquanto os que incluem os repositórios são oriundos das Ciências Físicas, Matemáticas, Sociais e Humanidades. Os autores desse relatório concluem que "os pesquisadores têm um conjunto de atitudes, percepções e comportamentos diante do sistema de comunicação científica e não desejam mudanças fundamentais na forma com que a pesquisa é atualmente publicada e disseminada" (FRY et. al, 2011, p. iv, tradução nossa). Também identificaram que os repositórios de acesso aberto são vistos corretamente como complementares à publicação convencional, ao invés de serem uma alternativa a esta. Cabe ressaltar que o objetivo destes repositórios não é e nunca foi, o de substituir o sistema atual de publicação científica (SUBER, 2012).

Este "conservadorismo" identificado entre os pesquisadores se relaciona com às mudanças tecnológicas no ecossistema de comunicação científica, que agora se sustenta nas TIC digitais. Mas é interessante observar que no estudo de Moore (2011), em Toronto, 87\% dos pesquisadores ouvidos concordam que os próprios pesquisadores precisam desenvolver um papel mais central em moldar e atuar no futuro do sistema de comunicação científica, ao passo que podemos inferir que aqueles do estudo europeu, PEER, entendem que este não seria tanto o papel deles próprios pois vêem a disponibilização em acesso aberto de artigos previamente publicados em canais fechados como não sendo da sua própria responsabilidade, mas de um intermediário, como, por exemplo, um bibliotecário ou repository manager (gestor de repositório de acesso aberto).

Em vários estudos, barreiras à maior adesão voluntária de pesquisadores às práticas e hábitos de acesso aberto são discutidos. Como já foi apontado, um resultado de todos as pesquisas feitas levantando as opiniões de pesquisadores é que eles majoritariamente concordam com os princípios, a ideia de acesso aberto. Assim, as explicações (barreiras identificadas) para a relativa baixa quantidade de material disponível em acesso aberto (no máximo 25\% do total mundial) englobam:

- Pouco conhecimento da questão e das implicações do acesso aberto; 
- Desinteresse no acesso aberto, como resultado da falsa concepção de que o acesso à literatura científica não é um problema para a maioria dos cientistas no mundo;

- Desconhecimento das revistas prestigiosas acesso aberto que já existem, devido ao hábito de publicar em, e ler, exclusivamente as revistas já conhecidos (a maioria de assinatura, não-acesso aberto), vistas como sendo mais seguras para motivos de progressão na carreira profissional;

- Desconhecimento dos repositórios de acesso aberto como forma de disseminar seus trabalhos, como forma complementar à publicação tradicional em revistas fechadas, pagas;

- $\quad$ Entendimento que depositar uma versão do trabalho já publicado num repositório de acesso aberto implica no autor infringir o copyright que cedeu à editora comercial;

- Percepção do procedimento de arquivamento como tecnicamente complexo e demandante de tempo precioso do pesquisador;

- Força do sistema de premiação na comunidade científica (academic reward system) pelo qual o prestígio pode contar muito mais que a disseminação rápida e ampla e pelo qual novas títulos de revistas entrando em cena ficam com uma relativa desvantagem diante dos títulos mais consolidados, e portanto, com maior reputação e prestígio.

\section{PROCEDIMENTOS METODOLÓGICOS}

Esta pesquisa pode ser caracterizada como descritiva, possuindo uma abordagem qualiquantitativa com predominância de métodos quantitativos (survey) para levantamento de dados, e formas mais qualitativas para análise dos dados quantitativos. Freitas et al. (2000, p.106) afirmam que a aplicação de um survey também é apropriada em pesquisas descritivas, pois este instrumento ajuda a "identificar quais situações, eventos, atitudes ou opiniões estão manifestos em uma população; descreve a distribuição de algum fenômeno na população ou entre subgrupos da população ou, ainda, faz uma comparação entre essas distribuições". O survey também é apropriado quando o fenômeno de interesse "ocorre no presente ou no passado recente" (FREITAS et al., op.cit. p. 105).

Foi utilizada a amostragem probabilística (aleatória simples), utilizando-se para fundamentar o cálculo do tamanho da amostra a base de dados de acesso público GeoCapes, que, no momento da aplicação do survey, mostrou que existiam 52.191 doutores permanentes distribuídos nas universidades brasileiras. Com um erro amostral de 5\%, nível de confiança de 99\%, o tamanho da amostra para esta quantidade de doutores foi de 643. Para conseguir este número de respondentes, tiveram que ser enviados um total de 3.340 convites por e-mail, explicando sucintamente o propósito do convite para participar do survey e fornecendo o link para o questionário, elaborado usando a plataforma livre LimeSurvey ${ }^{2}$, que permite também realizar análises estatísticas e manipulações básicas dos dados, bem como controlar e gerenciar convites, links e várias surveys simultâneas. Para que fossem os mais atualizados e confiáveis, os e-mails de contato dos pesquisadores foram colhidos "manualmente" de artigos publicados recentemente em revistas científicas disponibilizadas a partir do plataforma SciELO e de referências bibliográficas e abstracts disponíveis na Web of Science. O questionário do survey foi composto por 29 questões fechadas utilizando uma escala Likert, agrupadas em três grandes conjuntos que visaram captar o grau de conhecimento dos preceitos e práticas de acesso aberto detidos por pesquisadores, suas opiniões em relação ao ao, suas práticas e hábitos atuais em relação à publicação e uso de trabalhos científicos. O piloto da versão final do questionário foi testada (em versão impressa) com um conjunto de 12 pesquisadores, e comentários foram colhidos e incorporados na versão final. Os resultados da aplicação foram tabulados (usando o software Microsoft Excel) para serem visualizados e analisados, um sub-conjunto dos quais é apresentado na seção a seguir.

\section{DISCUSSÃO DOS RESULTADOS}

Os dados levantados no início do questionário para traçar o perfil da amostra

$2 \quad$ Disponível em: http://www.limesurvey.org/pt 
mostraram que $63,6 \%$ dos respondentes são do sexo masculino e $36,4 \%$ do sexo feminino. A distribuição dos respondentes por campo científico é apresentada na Tabela 1 abaixo. A estratificação por área e gênero mostrou uma distribuição relativamente equilibrada entre as áreas disciplinares: somente duas áreas tiveram um número notavelmente maior de respondentes do sexo masculino: as Ciências Exatas e da Terra (107 homens versus 22 mulheres) e as Engenharias (62 homens versus 11 mulheres).

Tabela 1 - A distribuição dos respondentes por área disciplinar

\begin{tabular}{c|c|c|c|c|c|c|c|c}
\hline Saúde & $\begin{array}{c}\text { Exatas \& } \\
\text { da Terra }\end{array}$ & Biológicas & Agrárias & Engenharias & Humanas & $\begin{array}{c}\text { Sociais Apli- } \\
\text { cadas }\end{array}$ & $\begin{array}{c}\text { Linguística, } \\
\text { Letras \& Artes }\end{array}$ & Outros \\
\hline $21 \%$ & $20,1 \%$ & $18 \%$ & $12,9 \%$ & $11,4 \%$ & $8,7 \%$ & $6,4 \%$ & $1,1 \%$ & $0,5 \%$ \\
\hline
\end{tabular}

Fonte: Própria

Um pouco mais que $90 \%$ dos respondentes concorda de alguma forma ou outra (total ou parcialmente) que o público em geral tem o direito de ter acesso aos artigos científicos resultantes de pesquisas financiadas dos cofres públicos. A pequena porcentagem $(1,9 \%)$ daqueles respondentes que discordam totalmente é constituída por pesquisadores das Ciência Agrárias, Ciências Exatas e da Terra.

Ao solicitar que os respondentes se posicionassem em relação a um enunciado sobre o conceito de acesso aberto, a saber: "Acesso aberto refere ao acesso gratuito a artigos científicos publicados em revistas científicas de acesso aberto, online e em repositórios de acesso aberto" as respostas, como vista na Tabela 2, demonstraram que a esmagadora maioria dos respondentes fica concentrada na categoria de resposta "Concordo totalmente". A estratificação por campo disciplinar, idade e gênero não indicou desvios ou correlações e associações.

Tabela 2 - Distribuição de concordância com o primeiro enunciado sobre acesso aberto

\begin{tabular}{|c|c|c|c|c|}
\hline $\begin{array}{c}\text { Discordo total- } \\
\text { mente }\end{array}$ & $\begin{array}{c}\text { Discordo parcial- } \\
\text { mente }\end{array}$ & $\begin{array}{c}\text { Nem discordo nem } \\
\text { concordo }\end{array}$ & $\begin{array}{c}\text { Concordo parcial- } \\
\text { mente }\end{array}$ & $\begin{array}{c}\text { Concordo total- } \\
\text { mente }\end{array}$ \\
\hline $1,9 \%$ & $1,9 \%$ & $0,8 \%$ & $18,2 \%$ & $77,3 \%$ \\
\hline
\end{tabular}

Fonte: Própria

A segunda questão procurou aprofundar este conceito "intuitivo" de acesso aberto, incorporando no segundo enunciado elementos presentes na definição da Budapeste Open Access Initiative - BOAI (2002) que explicita a questão dos direitos a reuso, cópia, redistribuição; ou seja, o conceito mais "forte". Na comparação com a questão, notou-se que a maioria dos respondentes permaneceu nas mesmas categorias, mas não foi um número insignificativo deles que mudou de opinião: de fato, $32 \%$ mudaram entre uma questão e outra, gravitando um pouco mais para o lado de discordância com o conceito de acesso aberto da BOAI.

Em relação ao conhecimento da existência de uma rede interoperável de repositórios de acesso aberto, mais que metade $(66,72 \%)$ sabe da sua existência, mas é relevante também observar que quase $33,28 \%$ (um terço) parece não ter certeza disso. Interessante observar também que a maior parte $(66,25 \%)$ dos respondentes está a favor do depósito de dados brutos neste tipo de repositório. Com a segmentação desse resultado por área, as Ciências Agrárias evidenciam a maior discordância com essa afirmação, sendo que $24,1 \%$ dos respondentes desta área discordam totalmente, comparado com o $37 \%$ dos seus pares que concordam totalmente, possivelmente apontando para esta área ser menos inclinada ao cenário de depósito de dados primários num repositório de acesso aberto. Entretanto, este cenário de disponibilizar dados 
de pesquisa em repositórios de acesso aberto talvez ainda seja hipotético para os respondentes, pois mais adiante no questionário foi perguntado se eles depositam, ou já depositaram ou depositariam tais dados, e a maioria $(68,2 \%)$ se encontra nas categorias de resposta de "nunca" ou "raramente".

O conhecimento sobre o fato de que o processo de revisão por pares se mantém e com o mesmo padrão de rigor para garantir a qualidade, tanto para artigos depositados em repositórios de acesso aberto, quanto para artigos publicados em revistas acesso aberto, foi revelado nas respostas às questões 12 e 13 . Quase $82 \%$ discordou com a colocação de que "Artigos depositados em repositórios de acesso aberto não passaram pela revisão por pares e é por isso que são disponíveis em repositórios de acesso aberto", e esta resposta segue a mesma distribuição nas respostas estratificadas por faixa etária e área. Quase metade dos respondentes $(48,83 \%)$ discordam totalmente com a crença de que "Os artigos disponíveis em revistas científicas acesso aberto passaram por uma revisão por pares menos rigorosa daqueles disponíveis nas revistas fechadas e comerciais", sendo que esses dois resultados demonstram uma forte convicção de que o fato do canal de disseminação ser acesso aberto não implica que os padrões de controle de qualidade sejam inferiores. Estas respostas também foram bem distribuídas por áreas de conhecimento. Contudo, as respostas sobre a passagem ou não por um processo de revisão por pares deste material encontrado nestes repositórios podem também apontar um desconhecimento do fato que é possível e aceitável depositar preprints nestes repositórios. De fato, esse é o cenário do arXiv e outros repositórios temáticos de acesso aberto, e é o que Harnad (2006) fortemente promove: que é possível ter $100 \%$ da literatura científica em acesso aberto "se e somente se" os autores a disponibilizarem imediata e simultaneamente ao enviar manuscritos à Editora. É neste momento de envio que o autor ainda detém o copyright total do trabalho, e o manuscrito - o preprint -sempre pertencerá ao autor.

De fato, os resultados desta pesquisa parecem apontar que existe confusão (esperada e identificada em outros estudos) em relação a copyright e o depósito em repositórios de acesso aberto. É interessante observar que, ao solicitar seu posicionamento em relação ao enunciado "Ao depositar num repositório digital uma cópia de um artigo já publicado numa revista científica, o autor está automaticamente infringindo as leis de copyright" a distribuição de cada categoria de resposta é quase igual, como mostra Gráfico 1. Tal resultado coincide com aquilo constatado na literatura, de que a questão de copyright fica uma área nebulosa: são poucos os autores que sabem que têm o direito de depositar versões dos seus artigos em repositórios de acesso aberto, dependendo da política de cada editora sobre a questão, que fica registrada, por exemplo, na base SHERPA-RoMEO (http://www.sherpa.ac.uk/ romeo/?la $=\mathrm{pt}$ ).

Gráfico 1 - Posicionamento em relação ao quesito de copyright de artigos num repositório de acesso aberto

Ao depositarnum repositório digital uma cópia de um artigo já publicado numa revista cientifica, o autor está automaticamente infringindo as leis de copyright.

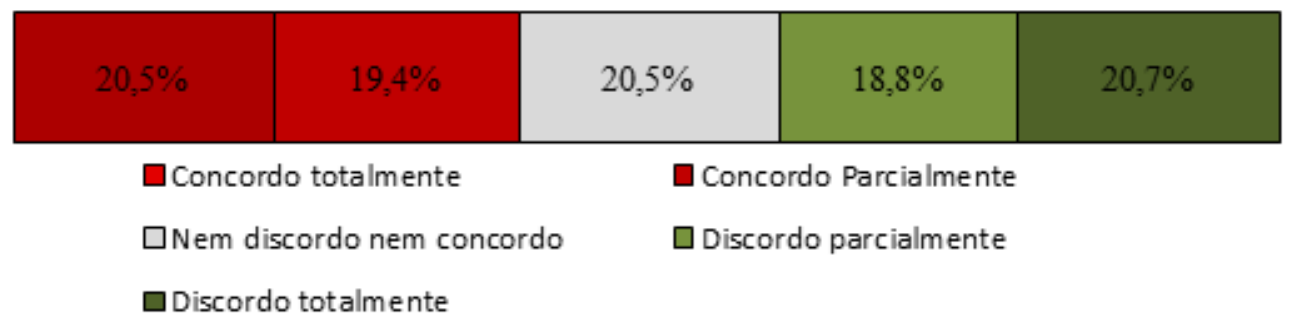

Fonte: Própria 
Mais duas questões procuraram traçar os hábitos dos pesquisadores de usarem repositórios de acesso aberto para o atual ou pretendido depósito de preprints e postprints dos seus trabalhos, e comparando as respostas das duas questões, detectamos nos resultados uma preferência pelo depósito dos postprints, ou a versão final do artigo, e de certa forma, uma aversão ao depósito do preprint, com 55\% afirmando que nunca deposita ou depositaria, e 14,5\% respondendo "raramente" para o depósito do preprint. Parece que para os respondentes, se um repositório de acesso aberto existe para disponibilizar os artigos científicos, é para disponibilizar somente as versões finais (postprints), que já passaram pelo processo de revisão por pares. Os respondentes com maior tendência de depositar uma versão de preprint num destes repositórios, são aqueles oriundos das Ciências Exatas e da Terra, com $48 \%$ dos respondentes desta área distribuída entre as categorias de respostas de "sempre", "frequentemente" e "às vezes". Possívelmente esta postura comparativamente um pouco menos "conservadora" em relação ao depósito de preprints dos pesquisadores das Exatas se deve à cultura mais arraigada do depósito e compartilhamento de preprints em repositórios de acesso aberto disciplinares e bem estabelecidos, como o famoso e prestigioso arXiv (lançado em 1991) da Cornell University. Interessante também observar que quando as respostas desta pergunta foram cruzadas com a variável de faixa etária, aqueles respondentes na faixa de 70-79 anos tinham mais probabilidade de responder "sempre", possivelmente indicando que os pesquisadores mais experientes são menos "preocupados" em depositar seus preprints, ou que se lembram da época em que preprints impressos eram livremente disseminados entre os pares científicos pelo mundo.

Em termos de hábitos de publicação entre os participantes, para o enunciado "Considero/aria a possibilidade de publicar meus trabalhos em revistas acesso aberto", os resultados, mostrados no Gráfico 2, revelam que $18 \%$ dos respondentes não consideraria publicar seus trabalhos em tais revistas, comparado com $42 \%$ que "sempre" e "frequentemente" considera tal publicação, além do $40 \%$ que respondeu "às vezes". Esta porcentagem de $40 \%$ para "às vezes" é significativa, porque poderia indicar que o fato de ser acesso aberto ou convencional não seja um critério que o autor-pesquisador usa para optar por um título para a submissão de artigos, ou poderia indicar desconhecimento de revistas/títulos em acesso aberto.

Gráfico 2 - Porcentagem de (possível ou atual) frequência de publicação em revistas acesso aberto.

\section{Considero publicar em revistas de Acesso Aberto:}

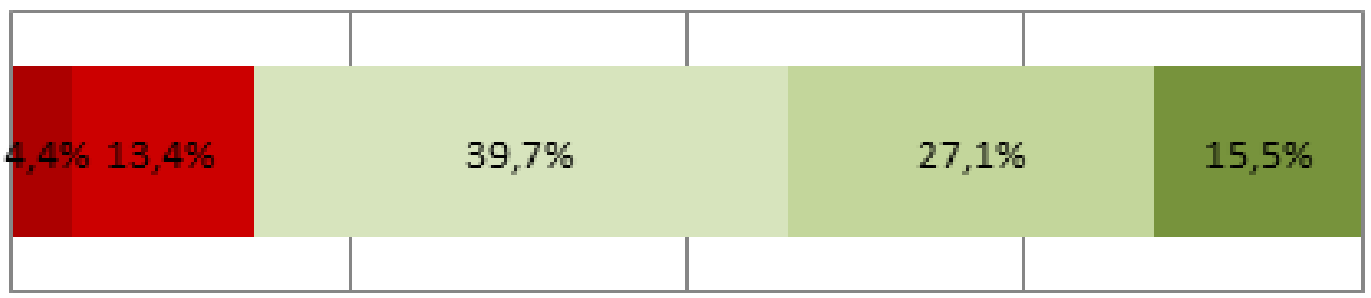

- Nunca Raramente Asvezes $\square$ Frequentemente $\quad$ Sempre

Fonte: Própria 
Foi significativo também que, ao se posicionarem diante do enunciado no questionário sobre a potencial vantagem de citação que os artigos disponibilizados em acesso aberto possam ter em relação aos canais fechados, a saber: "O Acesso Aberto às publicações científicas tem o potencial de aumentar o impacto destas, e assim, contribuir para a potencial evolução dos campos científicos", 53,7\% concorda totalmente, e 33,7\% concorda parcialmente.

Em termos de busca de informação, não foi surpreendente constatar que $75 \%$ das respostas ficaram distribuídas entre as categorias de "sempre", "frequentemente" e "às vezes" ao perguntar se os pesquisadores costumam utilizar o buscador Google Acadêmico (Google Scholar) para encontrar artigos na sua área disciplinar. As áreas de Saúde e as Biológicas mostraram menos probabilidade de se utilizar este ferramenta de busca. Usar o Google Acadêmico para encontrar artigos é de certa forma equivalente a buscar tais artigos em todos os repositórios de acesso aberto interoperáveis do mundo, ou seja, todos aqueles que funcionam de acordo com o protocolo OAI-MHP. Mesmo não tendo perguntando aos participantes se entram diretamente nos repositórios de acesso aberto, podemos inferir desse resultado que são usuários destes por conta dessa tendência do Google Acadêmico de se tornar o portal integrado de todos os repositórios de acesso aberto. No entanto, a já mencionada pesquisa PEER (FRY, et al., 2011) levantou que os tais repositórios não parecem estar muito no "radar" dos pesquisadores, que não os veêm como um recurso que possa trazer algo essencial para os pesquisadores, ou como algo que está faltando no sistema atual de comunicação científica. Mas os resultados das respostas a esta questão demonstram que, mesmo de forma inconsciente, os pesquisadores estão, de fato, utilizando repositórios de acesso aberto através do Google Acadêmico.

Ainda em relação ao seu comportamento de busca, acesso e uso da informação na forma de artigos, a grande maioria (91\%) já usou (leu e citou) artigos encontrados em acesso aberto. Os respondentes das Engenharias e as Ciências Exatas demonstram comparativamente a menor tendência de utilizá-los: da soma do total de respondentes que responderam "Nunca" (11) e "Raramente" (59), 40 respondentes são desses dois campos, ou seja, 57,1\% dos respondentes das categorias negativas. Há muita literatura das Ciências Exatas disponível em acesso aberto, principalmente devido à cultura das áreas desse campo de disponibilizar os resultados de pesquisa mais cedo no ciclo de publicação, utilizando repositórios de acesso aberto como o arXiv, por exemplo. Então, dentro desses respondentes (a 57,1\% acima citada), não podemos excluir a possibilidade de que leiam preprints e postprints encontrados em acesso aberto, mas que citem as versões publicadas em revistas de assinatura. Outra questão no survey procurou aferir este hábito de citação, e foi constatado que $63,5 \%$ dos respondentes cita sempre ou frequentemente a versão publicada numa revista convencional, de assinatura, mesmo tendo encontrado o material inicialmente num repositório de acesso aberto. Por fim, um respondente das Ciências Agrárias demonstrou sua adesão total ao princípio de acesso aberto, e alude, na sua colocação, à relevância de se utilizar canais abertos para aumentar o impacto potencial da pesquisa científica na sociedade como um todo: "Acredito que o acesso aberto é uma ótimo opção, pois já deixei de citar artigos que continham dados interessantes, devido à impossibilidade de acesso gratuito ao mesmo".

Houve uma questão que abordou o assunto de políticas obrigatórias de acesso aberto, requerendo que o respondente se posicionasse em relação ao enunciado "É errado as agências de fomento à pesquisa e/ou o governo e/ou as IES e os Institutos de Pesquisa obrigarem aos pesquisadores-autores disponibilizar seus artigos e produção científica em acesso aberto". Os resultados revelaram que quase 30\% discorda totalmente e $22 \%$ parcialmente com a ideia que não deveriam ser obrigados a disponibilizar sua produção científica em canais de acesso aberto; isto é, parece que esta parcela dos respondentes aceitaria mandatos, ou políticas obrigatórias para acesso aberto. Este resultado coincide com a postura favorável a mandatos de acesso aberto reportada nas pesquisas semelhantes. Swan (2006b) levantou dados em pelo menos dois estudos realizados com pesquisadores, que também constataram que a maioria dos respondentes afirma que cumpriria voluntariamente um mandato de auto-arquivar seus trabalhos num repositório institucional ou de acesso aberto. No entanto, como Swan (op. cit, 2006b) observa, é isso que os pesquisadores 
dizem que fariam: na prática, muitos repositórios de acesso aberto no mundo contém poucos artigos e manuscritos porque são poucas as IESs que fiscalizam e fazem cumprir os mandatos (institucionais e de agências de fomento).

A estratificação dos resultados (por faixa etária, gênero, ou área disciplinar) segue a mesma distribuição que a não estratificada, mas os respondentes das Engenharias demonstraram uma maior tendência de não concordarem com a obrigatoriedade para disponibilizar sua produção em acesso aberto, enquanto as Sociais Aplicadas e Humanas tendem na direção contraria, enquanto o resto da amostra se mantém equilibrada. Uma possível explicação desta tendência das Engenharias se sintonizarem menos com políticas obrigatórias de acesso aberto é que estas áreas produzem mais resultados sigilosos, com potencial aplicação no setor industrial, como um dos respondentes das Engenharias mencionou ao qualificar seu apoio pleno para o acesso aberto, no campo de respostas abertas: "O uso do acesso aberto em minha opinião pessoal não pode ser generalizado visto que muitas das pesquisas envolvem segredos industriais de seus investidores. No entanto, um sistema de acesso aberto, com a atual conjuntura de acesso a web facilitaria a divulgação do conhecimento e aceleraria o processo de desenvolvimento" (RespEng11)

A maioria dos comentários desse campo aberto no fim do questionário dizia respeito às percebidas limitações das revistas acesso aberto: são de "má-qualidade", os corpos editoriais são "questionáveis", são revistas "fakes" e "predatórias" (que enviam convites para publicar em e-mails não-solicitados), e isto diminui a sua credibilidade na percepção dos pesquisadores e potenciais autores. Evidentemente, esses comentários não se referem às revistas do SciELO, que não adotam tais práticas. É lamentável que uma minoria de revistas acesso aberto que seguem tais práticas duvidosas acabe "representando" a categoria inteira no imaginário dos pesquisadores.

\section{CONSIDERAÇÕES FINAIS}

Os resultados desta pesquisa com os pesquisadores da comunidade científica do Brasil apontaram que a grande maioria dos respondentes detêm conhecimento dos princípios e ações de acesso aberto, bem como manifestam apoio a estes. Foi identificado uma aceitação e adesão ao acesso aberto (mesmo que implícita, por vezes) em termos do seu comportamento informacional em relação à busca e uso (leitura e citação) da informação científica livremente disponível via canais de acesso aberto, e sobretudo, encontrando-a via o uso do buscador Google Acadêmico (que constitui, além de outras coisas, um portal à rede interoperável de repositórios de acesso aberto). Também revelam práticas e aceitação de publicação em revistas acesso aberto, mas sempre com a ressalva de que dada a oportunidade, optam pelos títulos com maior fator de impacto, resultado este em total consonância com pesquisas semelhantes realizadas em outros países, e que nos lembra que trata-se de uma comunidade de pessoas verdadeiramente "global" em termos de compartilhamento de valores comuns. As revistas com JIFs maiores fazem parte central do sistema de recompensa na comunidade científica que acaba constituindo um ciclo perpétuo que reforça o status quo dando maior valor para o artigo revisado pelos pares que aparece numa revista com alto fator de impacto. Mas podemos arriscar dizer que tal prestígio vale muito mais, para os participantes desta pesquisa, para o lado "produtor" da cadeia de comunicação, pois traz os benefícios profissionais na carreira acadêmica. Este fator parece não ter afetado sua percepção do uso de informação livremente disponível, encontrado via Google Acadêmico. Em alguns dos comentários individuais, muitos respondentes reclamaram da importância exagerada atribuída ao indicador fator de impacto, e apontam para as agências como CAPES e CNPq como tendo responsabilidade para reforçar tal importância.

Embora acessando, utilizando e publicando em acesso aberto, os respondentes pareceram demonstrar mais reservas no que diz respeito ao depósito (e/ou arquivamento) dos seus trabalhos em repositórios de acesso aberto (p.ex. em Repositórios Institucionais), e sobretudo, dos seus preprints (versão em manuscrito submetida à revista científica escolhida). Se a rede mundial e interoperável destes repositórios constitui uma "vitrine" para a pesquisa sendo desenvolvida e potencialmente publicada, os cientistas brasileiros mostram cautela em relação 
à divulgação e disponibilização imediata dos seus resultados e publicações nesse canal, embora também tenham demonstrado um alto nível de conhecimento das vantagens para seus grupos de pesquisa em termos da visibilidade advindas de tal disponibilidade imediata.

Esses resultados apontam a necessidade de focar o desenvolvimento de processos informativos e bem direcionados na promoção (advocacy) das ações institucionais e nacionais em torno do acesso aberto. Os pesquisadores precisam saber que podem depositar seus preprints, seus dados brutos da pesquisa, usar licenças abertas para garantir atribuição de autoria, consultar a base de SHERPA-RoMEO, e divulgar seus manuscritos sem comprometer a possibilidade destes serem aceitos na revista para qual foi submetido. De certa forma, tal tipo de pré-divulgação faria parte de políticas de acesso aberto (ou de mandatos), e os resultados também apontaram que a maioria dos participantes não são contra tais tipos de políticas, se forem instituídas pelos órgãos de fomento à pesquisa.

\section{Agradecimentos:}

Agradecemos o CNPq pelo financiamento concedido à primeira autora, processo $\mathrm{n}^{\mathrm{o}}$ 401875/2011-3

Artigo recebido em 0I/02/20I7 e aceito para publicação em I2/03/20I7

\section{BRAZILIAN RESEARCHERS VIEWS ON OPEN ACCESS TO SCIENTIFIC LITERATURE}

ABSTRACT: The research aimed to explore how various dimensions of open access to scientific literature is perceived by Brazilian researchers, also identifying their habits of publication, as well as use and citation of sources in open access. The methodological approach was quantitative, using a survey developed in the open software, Lime Survey, and consisting of 29 closed questions, responded by 643 permanent doctors from Brazilian universities distributed in all subject areas. Surveys completed by researchers from 3 I Institutes of Higher Education and Research Institutes in Brazil were received. Stratification by area showed a relatively balanced distribution between knowledge fields, with the largest percentage being from the Health Sciences (21\%), followed by the Physical and Earth Sciences (20.1\%) and the lowest percentage of $1.1 \%$ from Linguistics, Literature and Arts. The results indicate that the vast majority of participating researchers has knowledge of, and support for, open access principles and some projects promoting it, especially in relation to open access journal publishing, which also reflects in their usage habits and citation of such sources. Comparatively, the knowledge and use of open access repositories is smaller, and there seems to be some confusion regarding copyright status of archived articles in repositories. It was evident that the researchers believe that only postprints should be available in repositories. Ignorance identified regarding the compatibility between publication in "conventional" subscription journals, and deposit of the same article in open access repositories indicates the need for greater informative initiatives to clarify these aspects.

Keywords: Open access. Scientific researchers. Opinions. Scientific publication. communication. 


\section{REFERÊNCIAS}

BENKLER, Y. The idea of access to knowledge and the information commons: long term trends and basic elements. In: KRIKORIAN, G.; KAPCZYNSKI, A. Access to knowledge in the age of intellectual property. New York: Zone, 2010. p. 217-236.

BJÖRK , B.-C. et al. Open Access to the Scientific Journal Literature: Situation 2009. PLoS ONE 5(6), 2010. Disponível em: <http:/ / www.plosone. org/article/info\%3Adoi\%2F10.1371\%2Fjournal. pone.0011273>. Acesso em: 3 jan. 2014.

BONGIOVANI, P.; GÓMEZ , N. ; MIGUEL, S. Opiniones y hábitos de publicación en acceso abierto de los investigadores argentinos. Un estudio basado en los datos de la encuesta SOAP. Revista Española de Documentación Científica, v. 35, n. 3, julio-septiembre 2012. p. 453-467. Disponível em: < http://desarrollo.rephip.unr. edu.ar/handle/2133/1949>. Acesso em: 12 mar. 2013.

BUDAPEST OPEN ACCESS INITIATIVE. BOAI declaration. Budapest, 14 Fevereiro 2002. Disponível em: <http://www.soros.org/ openaccess/read>. Acesso em: 01 out. 2012.

CHALHUB, T.; RIBEIRO, L. V. P. Publicações de acesso livre: tendências entre pesquisadores de universidades públicas do estado do Rio de Janeiro. XII Enancib - Encontro Nacional de Pesquisa em Ciência da Informação. Brasília : ENANCIB. 2011. p. 2225-2241. 23 a 26 de outubro de 2011. Disponível em: <http:// repositorios.questoesemrede.uff.br/repositorios/ bitstream / handle / 123456789 / 2159/ Publica\%C3\%A7\%C3\%B5es\% 20-\% 20Chalhub. pdf?sequence=1>. Acesso em: 7 jan. 2013.

DAVIS, P.; CONNOLLY, M. Evaluating the reasons for non-use of Cornell University's installation of DSpace. D-Lib Magazine. v.13, n.3/4, 2007. Disponível em: <http://www.dlib. org/dlib/march07/davis/03davis.html>. Acesso em: 25 ago. 2011.

DAVIES, S. Analysis of Chemists and Economists survey on Open Access. Centre for Communications Research, University of Nottingham/JISC. Nottingham, 2011. Disponível em: <http://crc.nottingham.ac.uk/projects/rcs/ Chemists\&Economists_Analysis-Steve_Davies. pdf >. Acesso em:14 dez. 2013.

SANTOS, J. C. F. dos; MONTEIRO, S. A. Estudo da percepção de pesquisadores da área de ciências agrarias sobre acesso aberto. InCID: Revista de Ciência da Informação e Documentação, v. 4, n. 2, p. 34-53, jul./dez 2013.

FERREIRA, M. et al. Carrots and sticks: some ideas on how to create a successful institutional repository. D-Lib Magazine, v.14, n.1/2, 2008. Disponível em: <http://www.dlib.org/dlib/ january08/ferreira/01ferreira.html>. Acesso em: 15 out 2011.

FREITAS, H. et al. O método de pesquisa survey. Revista de Administração, São Paulo, v. 35, n. 3, p. 105-112, jul/set 2000.

FRY, J. et al. PEER behavioural research: authors and users vis-à-vis journals and repositories. Loughborough: LISU/Loughborough University, 2011. Disponível em: <http://www.peerproject. $\mathrm{eu} /$ fileadmin/media/reports/Final_revision_-_ behavioural_baseline_report_-_20_01_10.pdf>. Acesso em:

HAJJEM , C.; HARNAD, S.; YVES , G. Ten-year cross-disciplinary comparison of the growth of open access and how it increases research citation impact. arXiv preprints, 2006. Disponível em: <http://arxiv.org/pdf/cs.DL/0606079>. Acesso em: 3 abr. 2011.

HARNAD, S. Opening access by overcoming Zeno's paralysis. In: JACOBS, N. Open Access: 
key strategic, technical and economic aspects. Oxford: Chandos, 2006. p. 73-86.

MOORE, G. Survey of University of Toronto Faculty awareness, attitudes and practises regarding schloarly communication: a preliminary report. Toronto: University of Toronto, 2011. Disponível em: <https://tspace. library.utoronto.ca/handle/1807/26446>. Acesso em: 3 abr. 2011.

OpenDOAR. Growth of the OpenDOAR Database - Worldwide. 2017. Disponível em: <http://www.opendoar.org/onechart. php?cID $=\& c t I D=\& r t I D=\& c I I D=\& I I D=\quad$ \&potID $=\&$ rSoftWareName $=\& \quad$ search $=\&$ groupby $=r$. rDate Added \& orderby $=\&$ chartty pe $=$

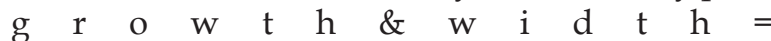
600\&height $=350$ \& caption $=$ Growth $\% \quad 20$ of $\% 20$ the $\% 20$ OpenDOAR $\% 2$ 0Database $\% 20-\% 20$ Worldwide>. Acesso em: 19 jan. 2017. Arquivado por WebCite ${ }^{\circledR}$ em: <http://www.webcitation. org/6ndLzk4lD>.

PARK, J.-H.; QIN, J. Exploring the willingness of scholars to accept open access: a grounded theory approach. Journal of Scholarly Publishing, v. 38, n. 2, Jan 2007. p. 55-84.

PROST, H.; SCHÖEPFEL,J. Degrees of openness: Access restrictions in institutional repositories. D-Lib Magazine, v.20, n. 7/8, July/August 2014. Disponível em: <http://purl.pt/302/1/dlib/ july14/prost/07prost.html>. Acesso em 23 julho, 2014.

RODRIGUES, E. Acesso livre ao conhecimento: a mudança no sistema de comunicação da ciência e os profissionais de informação. Cadernos de Biblioteconomia, Arquivistica e Documentação, v. 1, p. 24-35, 2004.

STONE, G. Report on the University Repository Survey. University of Huddersfield. Huddersfield, p. 10. 2010. Unpublished. Disponível em <http://eprints.hud. ac.uk/9257/>. Acesso em 11 nov.2013.

SUBER, P. Open Access. Cambridge, MA: MIT Press, 2012.
SWAN, A. Overview of scholoarly communication. In: JACOBS, N. Open access: key strategic, technical and economic aspects. Oxford: Chandos, 2006a. p. 3-12.

SWAN, A. The culture of open access: researchers' views and responses. In: JACOBS, N. Open Access: key strategic, technical and economic aspects. Oxford: Chandos, 2006b. p. 6572.

SWAN, A.; BROWN, S. Open access self-archiving: an author study. Key Perspectives, 2005. Disponível em: $<$ http://citeseerx.ist.psu.edu/ viewdoc/ download?doi=10.1.1.112.5048\&rep= rep1\&type=pdf>. Acesso em: 15 nov. 2009. 